\title{
Rekonstruktionen zum Verhältnis von Inklusionsverständnissen und -bedingungen in der Praxis von Lehrkräften
}

\section{Implikationen für die Entwicklung von Fort- und Weiterbildungen}

\section{Andreas Köpfer, Katharina Papke \& Jürgen Gerdes}

Journal für Psychologie, 27(2), 170-191

https://doi.org/10.30820/0942-2285-2019-2-170

www.journal-fuer-psychologie.de

\section{Zusammenfassung}

Innerhalb des bildungspolitischen wie wissenschaftlichen Fachdiskurses zeigt sich ein Bild höchst uneinheitlicher Verständnisse von Inklusion. Vor diesem Hintergrund stellt der vorliegende Beitrag die Frage, wie praktizierende Lehrkräfte den Reformauftrag schulischer Inklusion handlungspraktisch bearbeiten. Um dies exemplarisch zu beantworten, werden die impliziten Orientierungen von Lehrkräften auf Inklusion empirisch - mittels der Dokumentarischen Methode - rekonstruiert und an die schulisch-unterrichtlichen Bedingungen rückgebunden. Hierfür werden zunächst einige empirische Befunde vorgestellt sowie die theoretische Reflexionsfolie von »Inklusionsbedingungen « (Weisser 2017) dargelegt. Letztere dient als Bezugspunkt für die Sequenzanalysen der - im BMBF-geförderten Forschungsund Entwicklungsprojekt StiEL »Schule tatsächlich inklusiv« - erhobenen Interviews. Abschließend werden, vor dem Hintergrund der herausgearbeiteten Inklusionsverständnisse, erste Implikationen für die Fort- und Weiterbildungsgestaltung abgeleitet.

Schlüsselwörter: Inklusionsverständnis, Inklusionsbedingungen, Dokumentarische Methode, inklusive Schulentwicklung, Lehrkräftefortbildung

\section{Summary}

Reconstructions of the relationship between understandings and conditions of inclusion in the practice of teachers. Implications for the development of teacher trainings

In political and scientific discourse, highly heterogeneous understandings of inclusion exist. Against this background, the present article raises the question of how teachers process 
the inclusion agenda in their practices at school. Therefore, implicit teacher orientations are empirically reconstructed - using the documentary method of text interpretation - and related to the underlying conditions. For this purpose, some empirical perspectives on different understandings of inclusion are considered. The theoretical basis refers to so-called $\gg$ conditions of inclusion « (Weisser 2017), which are taken as reference points for the interpretation of sequence analysis of interviews conducted in the research and development project StiEL (founded by the German Federal Ministry of Education and Research). Finally, implications for a design of teacher trainings in inclusion are derived.

Key words: understanding of inclusion, conditions of inclusion, documentary method, inclusive school development, teacher trainings

\section{Einleitung - Thematisierungen von Inklusion in der Erziehungswissenschaft}

In den vergangenen Jahren hat sich Inklusion in Deutschland zu einem ubiquitären $>$ Buzzword < innerhalb gesellschaftlicher, wissenschaftlicher wie schulischer Diskurse entwickelt, wenn Phänomene der Teilhabe oder Separation in Bildungskontexten benannt und verhandelt werden. Dabei überlagern sich derzeit unterschiedliche Thematisierungen (vgl. Budde 2018). Beispielsweise wird mit Bezug auf die UN-Behindertenrechtskonvention (UN-BRK) (UN 2006) ein bildungspolitischer Reformdruck zur organisatorischen Umsetzung von Inklusion an die Schulen formuliert, der - aus menschenrechtlicher Perspektive verkürzt (Aichele und Kroworsch 2017) - mit der sukzessiven Verlagerung von Schüler*innen aus Förder- in Regelschulen verbunden wird. Gleichzeitig wird auf fachwissenschaftlicher Ebene ein Fokus auf die Entwicklung von (sonder-)pädagogischen Konzepten im Rahmen von Schul- und Unterrichtsforschung gerichtet, die primär den Blick auf Schüler*innen mit sonderpädagogischem Förderbedarf und deren inhaltliche wie soziale Teilhabemöglichkeit anvisiert. Wenngleich dabei oftmals Erweiterungen der Perspektive auf unterschiedliche Differenzlinien, wie zum Beispiel Migration, Sprache oder Geschlecht, sowie intersektionale Untersuchungen postuliert werden, verbleibt diese Thematisierungsfigur von Inklusion bei einer, zumeist askriptiv zugewiesenen, personengruppenbezogenen Perspektive (vgl. kritisch Köpfer 2019). Diese adressat*innenbezogenen Inklusionsverständnisse (oft wird hierbei von $>$ eng $<$ und > weit < gesprochen) lassen sich um eine strukturelle Ebene erweitern, womit eine Analyse der > behindernden < Strukturen in Organisationen fokussiert wird (vgl. ebd.). Im Anschluss daran beschäftigt sich ein weiterer Diskurs- und Forschungsstrang mit Fragen der Herstellung von Differenz in und durch Bildungsorganisationen bzw. durch deren Strukturen, Kulturen sowie Praktiken (vgl. Sturm und Wagner-Willi 2018). 
So zeigt sich innerhalb des erziehungswissenschaftlichen Fachdiskurses um $>$ Inklusive Bildung< ein höchst uneinheitliches Bild, was die Ebene der Adressat*innen sowie der Fokuspunkte für anstehende Transformationen anbelangt. Dies kann dabei nicht folgenlos für die handlungspraktische bzw. pädagogische Umsetzung von Inklusion bleiben (vgl. Trescher 2018), weshalb es gewinnbringend erscheint, die Inklusionsverständnisse der professionellen Akteur*innen im Feld Schule zu analysieren. Ein Blick in dieses Feld offenbart eine schulpraktische wie auch empirisch-analytische Problemlage: Zum einen arbeiten praktizierende Lehrkräfte in einem transformativen Feld, das derzeit von einem als >Inklusion < bezeichneten Reformdiskurs tangiert wird, der zwar administrativ und gesetzlich festgesetzt ist, aber - wie aufgezeigt - von unterschiedlichen fachlichen Einflüssen mit diversen Inklusionsverständnissen überlagert wird. Des Weiteren - und hier zeigt sich eine zweifache empirische Verkürzung - wird in der vor allem quantitativen Forschung zu inklusionsbezogenen Einstellungen von pädagogischen Fachkräften zumeist davon ausgegangen, dass Akteur*innen ein singuläres, statisches und widerspruchsfreies Inklusionsverständnis besitzen (kritisch hierzu Gasterstädt und Urban 2016), dessen sie sich bewusst sind und das sie explizieren können.

Dieser Beitrag intendiert daher, implizite Formationen von Inklusionsverständnissen in Gestalt von handlungsleitenden Orientierungen praktizierender Lehrkräfte empirisch zu rekonstruieren und diese an die strukturellen, kulturellen und handlungspraktischen Rahmenbedingungen rückzubinden. Hierfür werden zunächst einige empirische Befunde zu Inklusionsverständnissen angebracht sowie die theoretische Reflexionsfolie von »Inklusionsbedingungen« (Weisser 2017, 147) vorgestellt, die als Bezugspunkt für die darauffolgenden exemplarischen, mittels der Dokumentarischen Methode ausgeführten, Sequenzanalysen von Interviews mit Lehrkräften dient. Abschließend werden - vor dem Hintergrund der herausgestellten relationalen Inklusionsverständnisse - erste Implikationen für die Fort- und Weiterbildungsgestaltung formuliert.

\section{Empirische Befunde zu Inklusionsverständnissen}

In empirischer Hinsicht haben sich bereits eine Reihe von Wissenschaftler*innen mit Heterogenitäts- und Inklusionsverständnissen beschäftigt. So hat zum Beispiel Budde (2012) diskursanalytisch unterschiedliche Formationen der Thematisierung von Heterogenität in schulpädagogischen Diskursen herausgearbeitet. Darüber hinaus haben Piezunka, Schaffus und Grosche (2017) Expert*innen-Interviews mit Inklusionsforschenden aus unterschiedlichen Teildisziplinen (u. a. Disability Studies, Grundschulund Sonderpädagogik) geführt, um einen gemeinsamen Definitionskern herauszuarbeiten: Dabei wird eine Systematik aus vier Verständnissen - Inklusion im Sinne der 
UN-BRK, im Sinne der individuellen Leistungssteigerung von Schüler*innen sowie als Anerkennung bzw. als Utopie - entwickelt, deren Gemeinsamkeit darin verortet wird, Diskriminierung zu überwinden. Demgegenüber findet sich eine Rekonstruktion unterschiedlicher Inklusionsverständnisse bei Rotter und Schaaf (2015), die eine Dokumentenanalyse von Förderkonzepten auf Einzelschulebene unternehmen: Hier offenbart sich eine Spiegelung der Diskursbreite, die von einer Inklusionsinterpretation als Integration von Schüler*innen mit sonderpädagogischem Förderbedarf bis zu einer Adressierung aller Schüler*innen in ihren individuellen Besonderheiten reicht, wobei in der Tendenz eine Fokussierung auf erstere Gruppe bestehen bleibt.

Grundsätzlich gibt es jedoch nur wenige Untersuchungen (für einen Überblick vgl. Krischler et al. 2019) zu den Perspektiven der pädagogischen Fachkräfte. Eine Rekonstruktion der Akteur*innen-Sicht auf Inklusion findet sich bei Steiert (2017), die beschreibt, wie Schulsozialarbeitende ihre Inklusionsdeutungen auf die einzelschulischen Bedingungen herunterbrechen. Diese Analyse verbleibt jedoch bei der Feststellung eines Zusammenhangs zwischen den Rahmenbedingungen und den Perspektiven der Akteur*innen auf Inklusion, ohne näher zu charakterisieren, wie sich die Inklusionsverständnisse in den von den Lehrpersonen praktizierten »Inklusionsbedingungen « (Weisser 2017, 147) ausdrücken und in Rahmenbedingungen materialisieren.

So betonen etwa Gasterstädt und Urban $(2016,62)$ die Komplexität des Verhältnisses von Inklusionsverständnissen und Rahmenbedingungen - und die Bedeutung der Berücksichtigung dessen für eine Forschung über Inklusion. Daher sollten etwa Untersuchungen bezüglich der Einstellungen zu Inklusion eine explizite Betrachtung der zugrundeliegenden Verständnisse - und deren Entstehungsbedingungen - einschließen (vgl. für den internationalen Kontext auch Krischler et al. 2019). Eine genauere Betrachtung einzelschulischer Strukturen, Kulturen und Praxen dahingehend, wie bildungspolitische Vorgaben auf spezifische Weise, zum Beispiel durch Schulleitende, bearbeitet werden, erscheint somit zielführend. Einen vielversprechenden Ansatz bietet dabei eine Analyse, wie sie etwa von Budde et al. $(2014,107)$ zur Untersuchung von Inklusionsverständnissen in kommunalen Bildungslandschaften - am Beispiel eines Stadtfestes - genutzt wird: Hierin fällt ein besonderes Augenmerk darauf, wie sich diese aus den Verhandlungen zwischen den Akteur*innen und den institutionellen Gegebenheiten heraus konstituieren.

\section{Inklusionsbedingungen im Anschluss an Weisser (2017)}

Diesem Ansatz folgend fokussiert der vorliegende Artikel nicht das Ziel, eine einvernehmliche Definition von Inklusion herauszuarbeiten (hierfür siehe Piezunka et al. 2017), sondern empirisch Aufschluss darüber zu geben, wie sich bei dem pädagogischen 
Personal das Verhältnis von Inklusionsverständnissen und dem Feld zugrundeliegenden und fortlaufend praktizierten »Inklusionsbedingungen « (Weisser 2017, 147) artikuliert. Mittels letzterer bezeichnet Weisser, dass »in schulischen Bildungsverhältnissen [...] permanent Bedingungen gestellt [werden], deren Effekte als inkludierend respektive exkludierend beschrieben werden können « (ebd., 145). Diese weisen etwa einen Bezug zu den klassenstufenbezogenen Leistungserwartungen auf (vgl. ebd.). Im Falle der Unfähigkeit zur Erfüllung der Leistungserwartungen wird dabei versucht, »das Nicht-Erfüllen mit der Referenz auf > etwas $<$ zu verstehen und zu erklären « (ebd., 146). Weisser zeigt hierzu im Rahmen seiner Diskursanalyse, mit welcher relativen Beständigkeit - vom ausgehenden 19. Jahrhundert bis heute - Exklusionserklärungen im Schüler*innen-Subjekt verortet werden: dieses »ist nicht, hat nicht oder kann nicht « (ebd.). Im Zuge einer solchen Askription finden Entstehungsprozesse - etwa Dynamiken auf Basis sozialer Ungleichheiten - systematisch keine Beachtung (vgl. ebd., 149). Ermöglicht wird jedoch eine $\gg$ Teilung heterogener Schülergruppen entlang der Differenz von Normalität/Anormalität « (ebd., 53): Im Kreise des Verschiedenen kann so - über Defizitzuschreibungen - das Besondere (Anormale) abgespalten werden, wodurch graduelle zu kategorialen Unterschieden gemacht werden (vgl. ebd., 121). Eine solche kollektive Klassifizierung lebt davon, in ihrer Konstruktion auf (vermeintliche) Homogenität zu fokussieren und damit das Defizitäre zur alles dominierenden Personenbeschreibung zu erheben (vgl. ebd., 91):

»Kinder und Jugendliche, die der Schule beispielsweise mit einer Autismus-SpektrumStörung angekündigt werden, haben zunächst - wie alle anderen auch einen Vornamen und einen Namen und eine ihnen eigene Lern- und Entwicklungsbiografie. Dass daran erinnert werden muss, liegt an den Exklusionseffekten limitierter Inklusionsbedingungen: Diese determinieren die wechselseitige Wahrnehmung von Personen als Ganze und behalten die Anerkennung in aller Regel den inkludierten Personen vor « (Weisser 2017, 152-153).

Im Sinne einer Rechtfertigung schulischer Exklusionsprozesse müssen jedoch zunächst Anstrengungen dahingehend unternommen werden, die Defizite der Schüler*innen in ihren Ausprägungen transparent zu machen (vgl. ebd., 36). Dies ermöglicht schließlich die Festschreibung von Bedürftigkeiten sowie der hierauf antwortenden Behandlungsformen (vgl. ebd., 42). Dabei zeigt Weisser historisch auf, wie sich die Sonderpädagogik auf all jenes Wissen spezialisiert, welches zur Erklärung von abweichendem Verhalten und dessen Behandlung verwendet werden kann (vgl. ebd., 99). Dies beinhaltet die Schaffung differenzierter und individualisierter Bildungsangebote, die sich den Personen zugeschriebenen Merkmalen anpassen (vgl. ebd., 113), womit sukzessive eine moralische Dimension der Verantwortungsübernahme für Schüler*innen mit beson- 
deren Bedürfnissen bedient wird (vgl. ebd., 43). Hierdurch findet nicht zuletzt die Regelschule eine Entlastung, da diese folglich ihre grundsätzlichen Mechanismen von Ein- und Ausschluss nicht zu hinterfragen braucht (vgl. ebd., 114): »Das Problem ist, dass die Teilung zwischen allgemeiner und besonderer Erziehung und Bildung strukturell [Hervorheb. im Original] Möglichkeiten für Diskreditierung verfügbar hält und damit Bedingungen schafft, die eine partikulare, an unterschwelligen Vorstellungen von Normalität und Zugehörigkeit orientierte Kommunikation von Achtung/Missachtung legitimieren « (ebd., 153).

Insofern sind Zusammenhänge zwischen schulischen Bedingungen und Verständnissen von Inklusion beschrieben, welche im Weiteren an Hand empirischen Materials näher betrachtet werden.

\section{Datengrundlage: Interviews aus dem Forschungs- und Entwicklungsprojekt "StiEL - Schule tatsächlich inklusiv»}

Als empirische Datenbasis fungieren problemzentrierte Interviews (vgl. Witzel 2000) mit Akteur*innen schulischer Inklusion, die im Rahmen des BMBF-finanzierten Projekts »StiEL - Schule tatsächlich inklusiv « im Verbund der Pädagogischen Hochschule Freiburg, der Universität Potsdam sowie der Universität Bielefeld geführt wurden. Diese dienten dazu, die Akteur*innen zu den Gelingensbedingungen und Barrieren schulischer Inklusion zu befragen, um hierdurch die Möglichkeit zur Rekonstruktion ihres Handlungswissens zu erlangen. Der Datenkorpus umfasst insgesamt 134 Interviews mit Akteur*innen ${ }^{1}$ aus Baden-Württemberg, Brandenburg und Nordrhein-Westfalen, welche zunächst inhaltsanalytisch mit einem Fokus auf Fort- und Weiterbildungsbedarfe ausgewertet wurden. Die in den Interviews gewonnenen Daten werden exemplarisch im Rahmen dieses Beitrags vertiefender Analysen zum Thema der Inklusionsverständnisse praktizierender Lehrkräfte unterzogen. Auf der Basis dieser Ergebnisse werden im Projekt StiEL inklusionsbezogene Fortbildungsmodule für die Professionalisierung pädagogischen Personals entwickelt.

\section{Sequenzanalyse mittels Dokumentarischer Methode}

Leitend für die vertiefende Analyse ist die wissenssoziologisch fundierte Annahme (vgl. Mannheim 1980), dass sich Inklusionsverständnisse nicht vollständig kommunikativ explizieren lassen, sondern zum großen Teil vorbewusst sind und sich im Handeln der Akteur*innen dokumentieren (Bohnsack 2014). Daher werden ausgewählte Sequenzen der problemzentrierten Interviews (vgl. Witzel 2000) mittels der Dokumentarischen 
Methode (vgl. Bohnsack 2014) interpretiert, um hierdurch Zugang zu kollektiven Erfahrungen der Akteur*innen zu erhalten. So ist es das Ziel, Orientierungsmuster herauszuarbeiten, die zeigen, wie die Lehrkräfte den normativen Reformauftrag schulischer Inklusion vor dem Hintergrund vorzufindender Inklusionsbedingungen handlungspraktisch bearbeiten.

Hierfür wurden aus den Interviews Sequenzen mit besonders hohen narrativen Anteilen ausgewählt ${ }^{2}$, da diese der Handlungspraxis bzw. dem konjunktiven Erfahrungsraum der schulischen Akteur*innen besonders nahekommen (vgl. Nohl 2012) und sich in den Erzählungen zu konkreten Handlungssituationen die einzelschulischen Rahmenbedingungen verwoben finden. Diese Sequenzen wurden mittels des Transkriptionssystems TIQ (vgl. Przyborski und Wohlrab-Sahr 2014, 167ff.) bearbeitet und schließlich dokumentarisch interpretiert.

Ohne an dieser Stelle ausführlich auf das rekonstruktive Forschungsparadigma der Dokumentarischen Methode eingehen zu können (vgl. u. a. Bohnsack 2014; für schulische Inklusionsforschung Wagener 2018), werden kurz die zentralen Analyseschritte erläutert: Ziel ist es, sukzessive den für die impliziten Wissensstrukturen handlungsleitenden » modus operandi « (Bohnsack 2014, 256) der pädagogischen Fachkräfte zu erheben; das heißt, zu rekonstruieren, mit welchen Orientierungen die Akteur*innen ihre Handlungspraxis gestalten. Ein Orientierungsrahmen kann hierbei positiv, das heißt auf eine Orientierung zustrebend, oder negativ, von einem Ideal eines Sinnzusammenhangs abgrenzend, sein. Im Rahmen der Analyseschritte wird zunächst mittels Formulierender Interpretation (FI) der »immanente[n] Sinngehalt « (Nohl 2012, 41) des Explizierten im Transkript strukturiert. In der folgenden Reflektierenden Interpretation (RI) wird der Analysefokus dann vom »Was « auf das »Wie « des Gesagten geändert, um den » modus operandi « der Bearbeitung eines Themas zu ermitteln und somit die »konjunktive « Erfahrungsebene offenzulegen (vgl. ebd.). Die Komparative Sequenzanalyse dient schließlich der Herausarbeitung von Gemeinsamkeiten (Homologien) und Unterschieden (Heterologien) der Orientierung(en) innerhalb eines Falles bzw. zwischen unterschiedlichen Fällen, wie in den nachfolgenden Beispielen exerziert.

So werden an dieser Stelle drei exemplarische Rekonstruktionen zu Sequenzen aus Interviews mit einer Oberschul-Lehrkraft, einer Gymnasial-Lehrkraft sowie einer Realschul-Lehrkraft angeführt. Die Auswahl der Sequenzen aus dem umfassenden Datenkorpus erfolgte dabei entlang von Suchstrategien: Zum einen wurde in methodologischer Hinsicht nach dichten Erzählpassagen von Lehrpersonen gesucht; zum anderen wurde in thematischer Hinsicht nach Sequenzen gesucht, in denen der unterrichtliche (z. B. bzgl. Verhalten, Leistung, Curriculum) wie auch der schulische (z. B. bzgl. Kooperation, Schulorganisation) Umgang mit Heterogenität im Vordergrund steht. 


\section{Rekonstruktionen von Inklusionsverständnissen vor dem Hintergrund der Inklusionsbedingungen}

\section{1 "Junge aus der Grundschule»}

Die erste Sequenz stammt aus einem Interview mit der Lehrerin einer brandenburgischen Oberschule, welche sich in ihrer Selbstbeschreibung als > Schule für Gemeinsames Lernen< bezeichnet und über einen offenen Ganztagsbetrieb für die Sekundarstufe I verfügt. Im Folgenden handelt es sich dabei um die Anfangssequenz des Interviews, welche auf den thematischen Aspekt der multiprofessionellen Kooperation eingeht (vgl. Abb. 1).

1 Y1: Und das Aufnahmegerät is auch an; dann würde mich als (.) erstes

2 interessieren (.) wie ihnen Inklusion überhaupt in ihrem Arbeitsalltag

3 begegnet, was sie damit zu tun haben? genau. \#00:00:14.8\#

4 Af: Mhm (.) Inklusio:n ((atmet aus)) äh::m begleitet mich seit (.) mehren 5 Jahren (.) schon //mhm// und zwar hat unsre Grundschule (.) des Model Projekt 6 begonnen (.) inklusive Schule $/ / \mathrm{mhm} / /$ und äh:m zum damaligen Zeitpunkt 7 hab=ich auch an der Grundschule unterrichtet (.) insbesondere in einer Klasse (.) mit einem Jungen der (.) äh: lernbehindert ist //mhm// das ist jetzt so=n schönes Beispiel für Inklusion wo=se jetzt mal aufgetreten is ((atmet ein)) ansonsten sind ja=auch Schüler mit emotional sozialen äh::m (2) Schwächen nenn ich=s mal (.) mit dabei und (.) ja (.) die sind schon immer berufsbegleitend da gewesen //mhm// jetzt ham=se aber wirklich nen Namen odr und nen Titel bekommen //mhm// abr ansonsten (.) sind=se immer mit dabei gewesen; (.) und äh:m (.) diesen Jungen (.) aus der Grundschule den führ=ich jetzt in der neunten Klasse weiter //mhm// also (2) als Klassenlehrerin seit sieben (.) und insofern (2) kenn=ich den Jungen (.) mit seinen Einschränkungen $/ / \mathrm{mhm} / /$ (.) und äh:m (.) das Problem war in der Grundschule hat (.) war=s nen kleiner Kreis von Lehrer der mit ihm sich beschäftigen (.) musste //mhm// und mit Übergang in sie:ben war (.) ne komplett neue Lehrerschar da gewesen (.) di:e (.) erst=einmal unterrichtet werden musste (.) über den Jungen, seine (.) ja ich sach=mal seine Krankheitsbild beziehungsweise auch seine Vorgeschichte ((lässt die Hand auf den Tisch fallen)) //mhm// (2) große Unterstützung fand=ich (.) bei der (.) Sonderpädagogin (.) an unsrer Schule //mhm// weil sie=sich also auch bei den klinischen Bildern auskannte, mit den Fördermaßnahmen, öh welche Unterstützungsangebote müssen gegeben werden? welche Zusatzmaterialien müssen (.) geliefert werden $/ / \mathrm{mhm} / /$ oder bereit gestellt werden? insofern war sie eigentlich (.) die größte (.) Stütze und ist es immer noch //mhm// weil=s gibt halt och Lehrer die ( (atmet tief ein)) äh:m (2) bei denen geht=s einfach (.) im Alltag unter $/ / \mathrm{mhm} / /$ dass der Junge (.) gefördert werden muss (.) und äh:m (.) wir=ham jetzt auch das große Glück dass (.) er (.) ich will jetzt mal nich lügen (2) mit acht Stunden äh: : ähm (2) im Unterricht (.) im gemeinsamen Unterricht is, aber aus der Klasse rausgenommen wurde //mhm// äh:m ist ne kleine Gruppe (.) von fünf bis acht schülern die (.) die ähm bei ner Sonderpädagogin unterrichtet werden. \#00:02:34.3\#

Abbildung 1: Sequenz eines Interviews mit einer Oberschul-Lehrkraft (Brandenburg)

Auf den propositionalen Gehalt der Interviewerfrage, wie Inklusion überhaupt mit dem Alltag der interviewten Lehrkraft zu tun habe, validiert diese die Frage und elabo- 
riert sie, indem sie ihre mehrjährige Erfahrung mit Inklusion ausweist. Im Modus der Beschreibung referiert sie über die Zeit, als sie an der Grundschule unterrichtet hat. Sie fokussiert dabei auf einen Jungen, den sie als »lernbehindert « (Z.8) und zugleich als »schönes Beispiel für Inklusion « (Z.9) bezeichnet. Durch die explizite Einordnung als >tatsächliche Inklusion< (vgl. 9-12) eröffnet sich so ein Horizont der Bewertung von Inklusion bzw. Nicht-Inklusion, in den die Lehrperson zieldifferente Unterrichtung respektive zielgleiche Unterrichtung einordnet. Letztere sei bereits immer Teil des Schulalltags gewesen, wenn auch » $\underline{\text { berufsbegleitend }}$ (Z. 12). Damit wurde die Zuständigkeit in der Vergangenheit als nicht genuiner Bestandteil der eigenen Profession verortet - und nur provisorisch übernommen. Nun findet sich dieses - bislang improvisiert und diffus bearbeitete Phänomen - mit »Namen $\ll(Z .12)$ bzw. »Titel « (Z. 13) ausgestattet. Hier drückt sich ein positiver Horizont der Sichtbarmachung von Andersheit und - latent auch - der administrativen Kategorisierung klarer Zuweisung von Zuständigkeiten aus. Zudem offenbart sich eine Perspektive, in der Inklusion zunächst als Herausforderung für das professionelle Selbstverständnis vordergründig wird.

Durch die chronologische Beschreibung der Begleitung des Jungen während der Schulzeit deutet sich latent ein positiver Horizont der Beziehung im Rahmen einer erhöhten Kenntnis seiner Bedürfnisse an, der im Weiteren elaboriert wird. In der nachfolgenden Kontrastierung - »kleiner Kreis « (Z. 18) von Lehrkräften in der Grundschule versus »komplett neue Lehrerschar $\ll$ (Z. 19-20) mit Übergang in die siebte Klasse - eröffnet sich der Gegenhorizont eines Informationsbruchs sowie einer fehlenden Beziehung vor dem Hintergrund einer hohen Anzahl zuständiger Lehrkräfte. Dadurch wird die Orientierung der Lehrerin auf die Notwendigkeit des - über das $\gg$ Krankheitsbild « (Z. 21) - Informiertseins deutlich, welche durch die Betonung auf » Vorgeschichte (Z. 22) in ihrer Prozesshaftigkeit und Personenbezogenheit konkludiert wird. Das vollständige Informiertsein über das Kind deutet sich in der Perspektive der Lehrkräfte als notwendige Voraussetzung für pädagogisches Handeln an. Zugleich wird der Junge - mittels einer defizitorientierten Zuschreibung - zu einem Diskursobjekt, das über spezifische Informationen erklärbar, zugleich jedoch askriptiv verandert ${ }^{3}$ wird. Weisser $(2017,152)$ formuliert im Hinblick auf eine solche Diskursbildung zu Schüler*innen mit zugeschriebenen Förderbedarfen: »Sie sind Fremde, bevor sie als Person ihren Auftritt haben «.

Diesem Horizont wird schließlich eine einzelne Person gegenübergestellt, deren Handeln deutlich positiv - als »große Unterstützung « (Z. 23) - gerahmt wird. Die als »Sonderpädagogin « (Z. 24) betitelte Person wird im Modus der Argumentation als Informationsträgerin hinsichtlich des klinischen Bildes des Jungen eingeführt: So werden unterschiedliche Tätigkeitsbereiche (Fördermaßnahmen, Unterstützungsangebote, Zusatzmaterialien) elaboriert, für die sie als expertisiert angeführt wird. Die 
Tätigkeiten fokussieren dabei auf eine additive Förderung des Jungen. Durch die wiederholte Betonung der Unterstützung wird die Kontrastierung gegenüber den anderen Lehrern verstärkt. So deutet sich eine Orientierung der Interviewten auf das unterrichtliche Handeln der anderen Lehrkräfte an, welche eine Förderung und Differenzierung nicht als integralen Bestandteil des eigenen Unterrichts ansehen. Inklusion, im Sinne der Notwendigkeit der Förderung eines >lernschwachen< Jungen, wird also asymmetrisch und additiv zu den routinierten Praktiken der Lehrpersonen positioniert.

In diese Lücke tritt nun kompensatorisch die Sonderpädagogin und erscheint als mahnende Erinnernde bzw. Sichtbarmachende für Differenzierung und Förderung. Die Beschreibung kulminiert in der Darstellung des »große $[\mathrm{n}]$ Glück $[s] \ll$ (Z. 31), das darin besteht, dass der Junge für acht Stunden im gemeinsamen Unterricht sei und dort - außerhalb der Klasse - in einer kleinen Gruppe von Schüler*innen durch die Sonderpädagogin unterrichtet werde. Die explizit als »schönes Beispiel für Inklusion « (Z. 9) eingeführte Beschreibung bezieht sich so auf eine gelungene Förderung eines Jungen im Rahmen einer subsidiär-sonderpädagogischen Unterstützungsstruktur, die spezifische Förderung in und außerhalb des Klassenraums für diesen vorsieht. Vor dem Hintergrund des tendenziell - hinsichtlich Differenzierung - unveränderten unterrichtlichen Handelns der anderen Lehrkräfte wird eine Delegation des Jungen an das Unterstützungsangebot der Sonderpädagogin prozessiert.

\section{2 »Ohrschoner»}

Die zweite Sequenz entstammt dem Interview mit einer Lehrerin eines Gymnasiums in Baden-Württemberg. In ihrer Selbstbeschreibung zeichnet sich die Schule dadurch aus, Projekte zur Förderung besonders begabter Schüler*innen sowie zur humanistischen Persönlichkeitsbildung, ästhetischen Erziehung und zum sozialen wie personalisierten Lernen zu unterhalten. Diese Sequenz ist dabei ebenfalls dem Interview-Beginn entnommen und geht auf thematischer Ebene auf den Aspekt der Unterrichtsorganisation ein (vgl. Abb. 2).

Auf die Interviewfrage mit propositionalem Gehalt, wie der Lehrkraft Inklusion in ihrem Arbeitsalltag begegne, validiert sie diese mit Bezug darauf, dass an ihrer Schule »verstä:rkt Asperger Schüler « (Z. 5-6) aufgenommen wurden. Die Charakterisierung »Brennpunktschule « (Z.7) eröffnet dabei zu Beginn eine Orientierung auf konflikthafte Aspekte von Inklusion. Im Anschluss erfolgt eine Elaboration mit zwei Erzählungen über $>$ behinderte $<$ Schüler*innen. Die ausgewählte Sequenz führt die zweite Erzählung

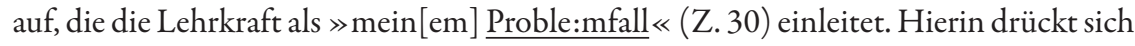
eine possessive Verbundenheit mit einem Problem aus, das sie in der Folge elaboriert. Zum einen verortet sie dieses in ihrem Unterrichtsfach (»Deutsch «, Z. 31) sowie in der 


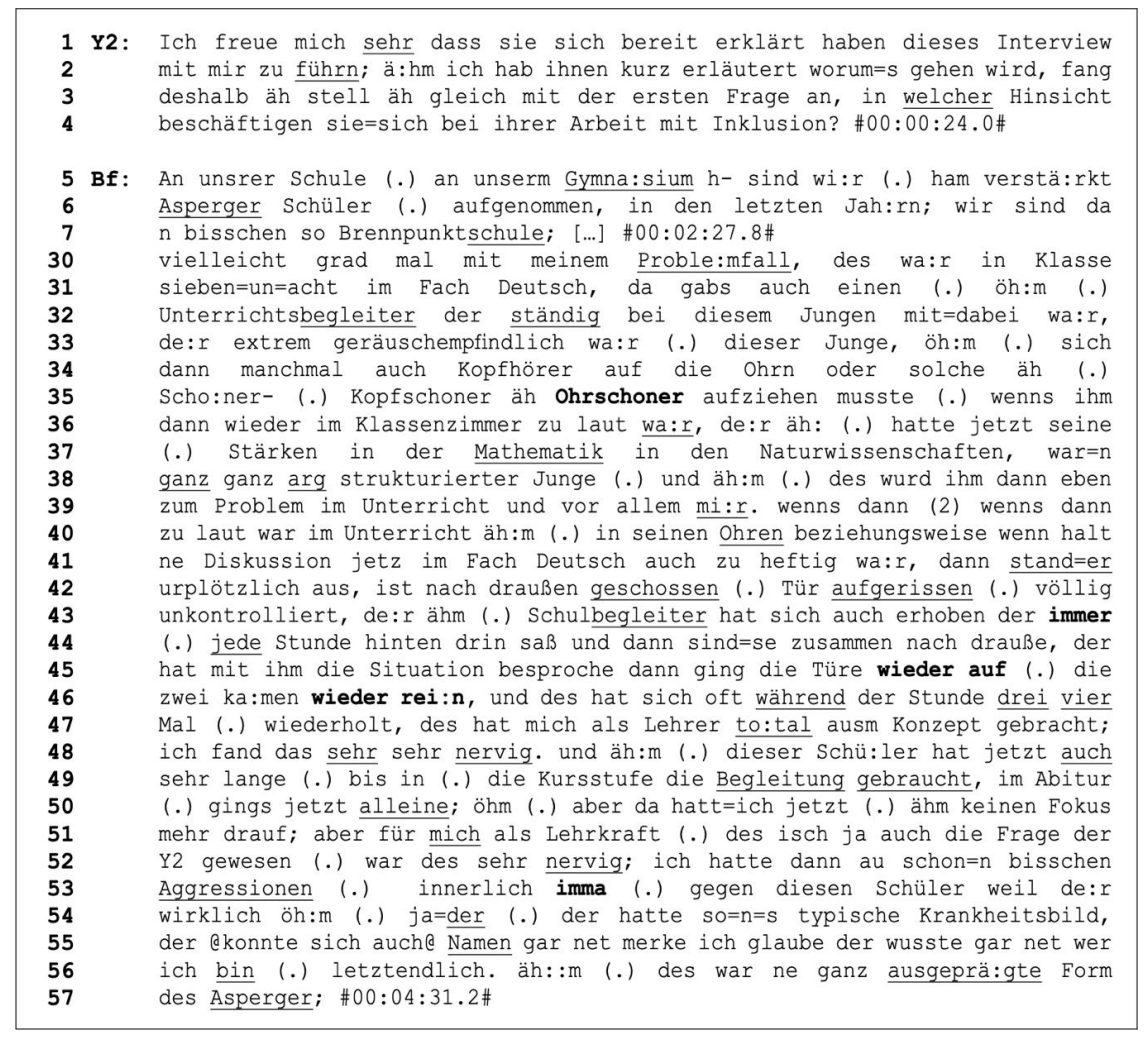

Abbildung 2: Sequenz eines Interviews mit einer Gymnasial-Lehrkraft (Baden-Württemberg)

Klassenstufe $\gg$ sieben=un=acht $\ll$ (Z. 31). Im Modus der Beschreibung führt sie einen Unterrichtsbegleiter ein, der sich ständig bei einem Jungen aufgehalten habe. Hierin klingt eine sukzessive Lokalisierung des Auslösers ihres Problems an, welches dann demonstrativ als »dieser Junge « (Z. 33) benannt wird. Insbesondere die Betonung der ständigen Präsenz des Unterrichtsbegleiters deutet auf einen permanenten und für sie nicht zu kontrollierenden Zustand hin, der entlang des >Schülermerkmals extremer Geräuschempfindlichkeit < weiter differenziert wird. In der darauffolgenden Beschreibung zum Verhalten des Jungen dokumentiert sich ein negativer Horizont der Nichtpassung von Geräuschempfindlichkeit und Unterrichtsgeschehen. Indem die Lehrkraft die personenbezogene kompensatorische Maßnahme - das Tragen von »Ohrschoner[n] « (Z.35) - wiederholt ausführt, emphasiert und elaboriert sie die situationale Nichtpassung als Problem des Jungen. 
Die Fokussierung auf kindspezifische Merkmale wird durch die Lehrkraft weiter elaboriert, indem nun ressourcenorientierte Aspekte, wie zum Beispiel mathematischnaturwissenschaftliche Stärken sowie ein hohes Maß an Strukturiertheit, aufgeführt werden. Hier eröffnet sich zwar ein latent positiver Horizont durch die Darstellung der Kompetenzen des Schülers; seine »Stärken « (Z. 37) finden jedoch deutlich geringere Beachtung - im Vergleich zur Attestierung der Nichtpassung im Hinblick auf die gängige Unterrichtspraxis. So kippt die Ressourcendarstellung (»ganz ganz arg strukturierter Junge «, Z. 38) postwendend in eine explizite Formulierung der Schwierigkeit: »des wurd ihm dann eben zum Problem « (Z. 38-39). Durch den Einschub (»und vor allem mi:r «, Z. 39) scheint diese Problemverortung jedoch nicht ohne Bezug auf ihre Person. Der positive Horizont wird damit durch die situationale Rahmung des Klassenraums gebrochen, wobei die Lehrkraft die Subjektivität der Laustärke-Empfindung deutlich herausstellt (»in seinen Ohren $\ll, Z$ Z. 40) - und damit eine leisere Unterrichtsgestaltung nicht zur Option bietet. Im Gegenteil verbindet sie die Lautstärke mit methodischen Aspekten des Unterrichts, indem sie im Modus der Erzählung elaboriert, wie der Schüler aufgrund heftiger Diskussionen im Fach Deutsch plötzlich und »völlig unkontrolliert « (Z.42-43) aufsteht und das Klassenzimmer verlässt.

Die Aus- und Wiedereingliederung in das Unterrichtsgeschehen - unterstützt durch den Schulbegleiter, dem eine ambivalente Rolle zukommt, da er einerseits als Teil der Störung eingeführt wird, er jedoch andererseits kompensatorisch im normalitätsorientierten Rahmen wirkt - bringt die interviewte Person in ihrer professionellen Rolle aus dem »Konzept « (Z. 47). Damit werden nicht zuletzt auch ihre Interessen - einer reibungsfreien Durchführung von Unterricht - in die Abwägungen zur $>$ Inkludierbarkeit< des Jungen eingeführt. Die Bewertung der Situation als »sehr sehr $\underline{\text { nervig } «(Z .48) ~ z e i g t ~ a n, ~ w o r a n ~ s i c h ~ d a s ~ E r g e b n i s ~ o r i e n t i e r t: ~ a n ~ e i n e r ~ B e i b e h a l t u n g ~}$ unterrichtlicher Fachpraxis, die die Erwartung einer Anpassungsleistung - mittels kompensatorischer Medien wie Ohrschoner, Begleitung und beruhigende Gespräche - an den Schüler adressiert. Die Erfüllung dessen scheint für die Lehrkraft eine basale Inklusionsbedingung darzustellen. So erfährt etwa das bestandene Abitur keine gesonderte Elaboration oder Bewertung - im Gegensatz zu den Exklusionsprozessen.

Die Thematisierung wird zum Abschluss rituell konkludiert, indem die Lehrkraft die Fragestellung der Proposition verschiebt - von den Ausprägungen ihrer professionellen Beschäftigung mit dem Thema Inklusion hin zu den Ausprägungen ihrer persönlichen Empfindungen (vgl. Z. 3-4 und Z. 51-52). Damit drängt der Aspekt unterrichtlicher Ordnung als prioritärer - weil von der Lehrperson zu bewältigender - in den Vordergrund, der das >Problem< des Jungen überlagert. Vor dem Hintergrund dessen, dass sie diese Problembewältigung nicht als ihre professionelle Aufgabe kodiert - sondern etwa als die des Schulbegleiters, kann sie ihre »Aggressionen « (Z. 53) frei zum Ausdruck bringen. Dabei ermöglicht die Kategorisierung »typische[s] Krank- 
heitsbild « (Z. 54) die Artikulation der Besonderung und verstärkt die Legitimierung des $>$ Otherings $<$. So scheint die Ontologisierung des Jungen entlang typischer Merkmale von Autismus, ein defizitorientiertes Sprechen zu befördern (vgl. Pfahl 2011), welches den Kontrollverlust der Lehrkraft als permanentes Defizit des Schülers fasst. Mittels der Projektion auf die Behinderung (»ne ganz ausgeprä:gte Form des Asperger $\ll$, Z. 56-57) wird eine deutliche Distanzierung prozessiert ( $\gg$ der wusste gar net wer ich bin «, Z. 55-56): so deutet sich hierin zwar der Horizont einer gewünschten Beziehung zu dem Schüler an, gleichzeitig jedoch werden die Gründe für das NichtZustandekommen ausschließlich im Krankheitsbild und nicht in der situationalen Rahmung - etwa der Lautstärke im Klassenraum - gesehen.

\section{3 »Digitales Klassenbuch»}

Die letzte Sequenz ist dem Interview mit einer Lehrerin entnommen, die an einer Realschule in Baden-Württemberg unterrichtet. Die Schule charakterisiert sich durch einen >offenen Ganztagsbetrieb < sowie eine spezifische Förderung von Formen des selbstorganisierten Lernens mittels Lernwerkstätten. Im Rahmen dieser Sequenz - aus dem Mittelpart des Interviews - vertieft die Befragte Ausführungen zu einem schuleigenen »digitalen Raum « (Z. 266) und damit zu Aspekten der Schulorganisation (vgl. Abb. 3).

Auf die beschreibungsgenerierende Frage der interviewenden Person, ob die Lehrkraft den zuvor von ihr erwähnten »digitalen Raum « (Z. 266) näher erläutern bzw. dessen Funktionen ausführen könne, elaboriert diese, es handle sich dabei um ein » $\underline{\text { di- }}$ gitale $[s]$ Klassenbuch « (Z. 269). Der neutrale Ausdruck >Raum < wird so durch eine Begrifflichkeit mit klarer Zugehörigkeit zum Kontextfeld Schule ersetzt. Dies wird weitergeführt, indem die Lehrkraft im Modus der Beschreibung den Zusammenhang zum Vertretungsplanprogramm elaboriert und damit die Verknüpfung mit der schulischen Routine aufzeigt. Sie führt dann aus, dass der Stundenplan wie auch das Klassenbuch in Form einer $\gg$ App « (Z.272) vorliegen, wodurch sie dieses - mittels ihres Handys - » zum Beispiel mit hoch « (Z. 271) nehmen könne. Damit emphasiert die Lehrkraft eine gewisse Weitläufigkeit des Schulgebäudes, die offenbar eine Notwendigkeit für effiziente Lösungsansätze der Schulorganisation schaffe. Ihre Elaboration gewinnt an dieser Stelle argumentativen Charakter: So könne sie etwa für jede Stunde eintragen, welcher »Lernstoff « (Z. 274) durchgenommen wurde, womit die Nützlichkeit der App nochmals herausgestellt ist. Hierauf aufbauend führt die Lehrkraft die Zwischenkonklusion an, das $>$ digitale Klassenbuch $<$ sei $\gg$ wie $=$ n normales Klassenbuch $\ll$ (Z. 274-275).

Dabei zeigt die Weiterführung »kann=aber auch « (Z. 275), dass dessen Funktionalität offenbar nicht in der eines normalen Klassenbuches erschöpft ist: So könne sie etwa für jede*n ihrer Schüler*innen »Einträge « (Z. 276), »Vermerke « (ebd.) und 
266 Y2: Können sie diesen digitalen Raum nochmal beschreiben //mhm// was da genau 267 äh:m //mhm// abgelegt werden kann (.) oder wie die Funktionen sind? \#00:19:11.4\#

269 Cf: Genau also des digitale Klassenbuch des (.) äh:m hängt auch mit unsrem dann halt so ab (.) ähm ich n- nehm mein Handy zum Beispiel mit hoch (.) hab die App unsres äh:m Stundenplans (.) und äh:m (.) ja (.) des Klassenbuch is im Prinzip auf der App auf der Handy-App (.) und kann=dann äh:m (.) für jede Stunde eintragen, was hab ich an Lernstoff gemacht? also als (.) wie=n normales Klassenbuch in dem Sinne ((atmet aus)) kann=aber auch bei jedem Schüler zum Beispiel Einträge Vermerke Notizen (.) und so weiter machn, kann aber=auch äh bei den Hausaufgaben zum Beispiel sagen (.) ah: diese Hausaufgaben sind nur für (.) Julia und Caitlin, diese Hausaufgaben sind für den Rest der Klasse; also ich kann in dem Sinne schon sehr=viel kommuniziern. und der Klassenlehrer (.) und auch die Inklusionslehrerin die haben (.) alle Zugriff auf diese Schüler, also die Schüler unsrer Klasse können dann von den entsprechenden Lehrkräften auch eingesehen werden //mhm// und da sieht man dann auch relativ schnell (.) ah was is wieder vorgefallen? und das Gute is, deswegen ham=wir dieses digitale Klassenbuch auch dieses Jahr eingeführt (.) des ist nicht nur für uns Lehrer sondern auch für die Da:men aus der (.) Diakonie die dann nachmittags zum Beispiel auch eintragen können (.) Julia und Caitlin (.) flippen (.) im Saa:l total aus und dann können wir auch wirklich reagieren $/ / \mathrm{mhm} / /$ und des war so der Knackpunkt letztes Jahr bei uns in der Ganztagsbetreuung dass wir diese Übersicht nicht hatten, mh uns ist teilweise abhanden gekommen warn die Kinder da:? warn=se nicht da? was ist da eigentlich passiert? ähm (.) welche Maßnahmen muss ich ergreifen? und=so=weiter. und das is des gro: Be Ziel eigentlich auch gewesen dieses digitalen Klassenbuchs dass=wir (.) mehr Austausch mitnander haben (.) //mhm// genau. \#00:20:44.5\#

Abbildung 3: Sequenz eines Interviews mit einer Realschul-Lehrkraft (Baden-Württemberg)

»Notizen « (ebd.) machen. Die Elaboration der Lehrkraft changiert so in Bezug auf das digitale Klassenbuch zwischen der Herstellung einer Anbindung an schulische Normalität und der Herausstellung eines zusätzlichen Nutzwertes: Betrachtet man die Ausführungen, so lassen sich » Notizen $\ll(Z .276)$ eher weniger in das klassische Schulvokabular einreihen. Stehen $>$ Einträge $<$ mit Verstößen - das heißt einem bestimmten und zunächst einmal singulären Ereignis von gewisser Schwere - in Verbindung, so wohnt dem Begriff >Notiz< eine Routinemäßigkeit inne. Darüber hinaus bestehen im schulischen Rahmen - etwa der Schulordnung - gewöhnlich Regularien zum Gebrauch von Klassenbucheinträgen: so müssen diese in der Regel an die Schüler*innen kommuniziert werden, was die Möglichkeit impliziert, sich gegen - aus der Sicht von Schüler*innen - ungerechtfertigte Disziplinarmaßnahmen zur Wehr zu setzen. Ist hierin die Potenzialität eines Dialoges zwischen Schüler*innen und Lehrkräften angelegt, beschreiben >Notizen < eher monologische Kommunikationen auf Basis eines routinemäßigen Erfassens von Schüler*innen-Verhalten.

Im Weiteren elaboriert die Lehrkraft exemplifizierend, dass das >digitale Klassenbuch< auch die Möglichkeit biete, festzuschreiben, welche Hausaufgaben »nur für (.) 
Julia und Caitlin « (Z. 278), und welche für »den Rest der Klasse« (Z. 279) sind. Die Besonderung der beiden Schüler*innen, die nicht zum $>$ Rest der Klasse $<$ gehören, wird so mittels der personenbezogenen, kompensatorischen Maßnahme individualisierter Hausaufgaben prozessiert. Hierauf führt die Lehrkraft erneut eine Zwischenkonklusion an, die besagt, sie könne mittels des >digitalen Klassenbuches $<\gg$ schon $\underline{\operatorname{sehr}=v i e l}$ kommuniziern « (Z. 279): So hätten die zuständigen Lehrkräfte »alle Zugriff auf diese Schüler « (Z. 280-281), was bedeute, diese können von den Lehrkräften »eingesehen « (Z. 282) werden. Als Grundorientierung lässt sich damit herausstellen, dass Kommunikation einseitig - und nicht dialogisch - gefasst wird und in erster Linie auf Kontrolle abzielt. So wird von der Befragten kein Horizont des Austauschs, der Verständigung und gegenseitigen Beratschlagung der Lehrkräfte eröffnet, sondern es scheinen vielmehr eindeutige Sachlagen vorzuliegen, die dies nicht erforderlich machen. In der Verwendung des Wortes »Zugriff « (Z. 281) deutet sich zudem die Einschätzung einer Notwendigkeit zur $\gg$ schnell[en] « (Z. 283) Kontrolle an, die mit der Frage in Verbindung steht: » was is wieder vorgefallen? « (Z. 283). Es wird von der Lehrkraft positiv (»das Gute«, Z. 283) - und als Grund für die Einführung des >digitalen Klassenbuchs < - gewertet, dass auch in der Nachmittagsbetreuung Eintragungen zu den Schüler*innen vorgenommen werden können (vgl. Z. 286). Das Exempel der Elaboration fällt dabei ein weiteres Mal auf die im Klassenverband bereits besonderten Personen: »Julia und Caitlin (.) flippen (.) im Saa:1 total aus « (Z. 287). Hierbei wird das Verhalten der beiden Schüler*innen als Legitimation für die Verfahrenspraxis - des kontrollierenden >Zugriffs $<-$ herangezogen; es wird jedoch nicht elaboriert, wie sich die Handlungssituation im Detail ausgestaltete. Überlegungen zu Ursachen und Ausführungen zu den Rahmenbedingungen des Verhaltens der beiden Kinder finden so keinen Raum, wodurch sich ein nahezu > dämonisierender< Diskurs in Bezug auf Julia und Caitlin herausbildet, der die Gründe für ihr Verhalten auf ihre Personen zurückführt. Hierbei wird deutlich, wie die Schaffung von Zugehörigkeit bzw. NichtZugehörigkeit ein achtendes bzw. missachtendes Sprechen legitimiert (vgl. Weisser 2017, 153).

Kontrollierbarkeit von Verhalten wird in diesem Sinne zu einer basalen Inklusionsbedingung, bzw. es gestaltet sich als » Knackpunkt « (Z. 288), wenn die »Übersicht « (Z. 289) - und damit die Möglichkeit zur Kontrolle - »abhanden « (Z. 290) kommt. Hierbei können die pädagogischen Fachkräfte dann »wirklich reagieren « (Z. 288), wenn sie alle $>$ Vorfälle $<$ kennen, während tiefergreifende Auseinandersetzungen mit dem Verhalten und dessen Bedingungen scheinbar nicht notwendig für das Ergreifen adäquater »Maßnahmen « (Z. 291) sind. Zum Abschluss kommt es zu einer rituellen Konklusion, in dem Sinne, als dass die Befragte von der Ermöglichung von $\gg$ mehr Austausch « (Z. 293) durch das > digitale Klassenbuch < spricht, jedoch nicht thematisiert - und damit ausklammert, wie sich dieser $>$ Austausch $<$ zwischen dem päd- 
agogischen Personal gestaltet, bzw. ob damit tatsächlich wechselseitige Interaktionen zur Verständigung und Beratschlagung oder einseitige $>$ Zugriffe $<$ mehrerer Personen in Bezug auf einen Sachverhalt gemeint sind.

\subsection{Komparative Analyse}

Vor dem Hintergrund der vorangegangenen Interpretationen werden in komparativer Analyse der Orientierungsrahmen der Lehrkräfte Homologien und Heterologien sichtbar, die zunächst bzgl. der Inklusionsverständnisse, im weiteren Verlauf dann bezogen auf die hierdurch prozessierten Inklusionsbedingungen skizziert werden.

\section{Inklusionsverständnisse}

Zum einen zeigt sich ein kollektiver Orientierungsrahmen dahingehend, als dass die drei Lehrkräfte Inklusion als professionell zu erfüllenden Auftrag zur personenbezogenen Integration von Schüler*innen mit zugewiesenem sonderpädagogischen Förderbedarf in ihrem Unterricht bearbeiten. Es handelt sich demnach um einen > von außen< an sie gerichteten Auftrag, den sie erfüllen, indem sie die Schüler*innen als gelabelte Diskursobjekte (re-)produzieren und deren Abweichung als Anlass für Interventionen in ihr schulisch-unterrichtliches Handeln fassen.

Weiter zeigt sich eine latente Orientierung bezogen auf Inklusion dahingehend, als diese im Sinne einer herausfordernden Aufgabe an das professionelle Selbst bearbeitet wird (vgl. Abschnitt 6.1). Dies drückt sich in Abwägungen hinsichtlich der eigenen Bewältigungsfähigkeit sowie des Arbeitsaufwandes aus. Damit ordnen die Lehrkräfte den Auftrag zur Inklusion - bzw. in ihrem Sinne zur Integration von Schüler*innen mit sonderpädagogischem Förderbedarf - in Relation zu den eigenen institutionellen, professionellen und kooperativen Rahmenbedingungen ein. Hierbei zeigt sich - im Rahmen der drei angeführten Analysen - das Inklusionsverständnis der Lehrkräfte dahingehend homolog, als dass sie damit das latente Ziel der - ggf. sonderpädagogisch unterstützten - Einpassung in den bestehenden Normalitätsrahmen verbinden. So offenbart sich hier eine Nähe zum Verständnis von Inklusion, das die Fördernotwendigkeit einzelner defizitärer Schüler*innen in den Mittelpunkt rückt.

Zugleich finden sich jedoch auch »Inklusionsbedingungen « (vgl. Abschnitt 3) in Form situationaler >Auflagen < für die abweichenden Schüler*innen. Vor dem Hintergrund eines grundsätzlich von einer Leistungsnorm, die mit korrespondierenden Praktiken der Bewertung und Selektion einhergeht, dominierten Feldes kann dies nicht als Veränderungsresistenz der Lehrkräfte gegenüber ihrer eigenen Praxis, sondern letztlich als konsequent erwartungskonformes Handeln angesehen werden. So zeigen 
sich konsistent kollektive Orientierungen der Lehrkräfte in Bezug auf eine personelle Auslagerung der Förderung vor dem Hintergrund dessen, dass im Rahmen sonderpädagogischer Maßnahmen eine Passung von >Krankheitsbild< und Fördermaßnahme verortet wird, womit im Umkehrschluss an den grundlegenden regelschulischen Mechanismen von Ein- und Ausschluss festgehalten werden kann (vgl. Weisser 2017, 114; Abschnitt 3).

\section{Inklusionsbedingungen}

Die vorangegangenen Rekonstruktionen zeigen, dass die Inklusionsverständnisse rückgebunden sind an die Grundkonstitution eines von Selektion geprägten Feldes, mit dem die Lehrkräfte auch gerade deshalb nicht in Konflikt treten (können), da sie durch sonderpädagogische Leistungen von einer eigenen Verantwortungsübernahme moralisch entlastet werden (vgl. ebd., 43; Abschnitt 3). Vielmehr artikulieren sich durch die explizit inklusionsbezogenen Erzählungen der Lehrkräfte immanente Handlungsstrukturen und -kulturen, die - über die bereits bestehenden institutionell-rechtlichen Zugangsbedingungen der jeweiligen Schulform hinaus - implizite, situationale »Inklusionsbedingungen « zum Ausdruck bringen, welche innerhalb eines >inklusiv < ausgewiesenen Feldes als Exklusionspraktiken sichtbar werden. In den Rekonstruktionen offenbart sich also eine Eingebundenheit der Akteur*innen in eine Praxis, die den Schüler*innen - insbesondere leistungs- und verhaltensbezogene - Bedingungen zur Teilhabe auferlegt. Diese dokumentieren sich auf einer impliziten Ebene in den Praktiken der Lehrkräfte und sind für die Beobachtung erster Ordnung unsichtbar; sie werden nicht direkt (explizit) an die Schüler*innen vermittelt, sondern finden sich invers in Exklusionspraktiken des Schulteams. Somit können sich die Schüler*innen nicht >ad hoc< dazu äußern.

Die vorangegangenen Rekonstruktionen machen deutlich, dass Inklusionsbedingungen sowohl auf der unterrichtlichen wie auch der schulischen Ebene angesiedelt sind:

$>$ Unterricht (vgl. Abschnitt 6.2): In Relation zu den leistungs- und verhaltensbezogenen Rahmenbedingungen sowie zur eingeschätzten eigenen Professionalisierung (z. B. im Umgang mit Störungen) und zur Bereitschaft für Differenzierungsaufwand werden implizit situationale Inklusionsbedingungen durch die Lehrkraft vermittelt (z. B. Stillhalten bei lautstärkebedingt normierten Diskussionsrunden, Aufsicht durch Schulbegleitung).

$>$ Schulorganisation (vgl. Abschnitt 6.3): Hier zeigt sich etwa die Inklusionsbedingung einer bürokratischen Informiertheit über abweichendes Verhalten durch digitale Überprüfung. Mittels einer dauerhaften Verhaltenskontrolle werden die >Vorfälle auf den Konten< der Schüler*innen erfasst, um entspre- 
chende Maßnahmen einzuführen. Dabei bilden die Schüler*innen mit zugeschriebenem sonderpädagogischen Förderbedarf eine von vornherein gesonderte Beobachtungsdimension.

Homolog sind die rekonstruierten Sequenzen darin, dass eine Inklusionsbedingung die Notwendigkeit sonderpädagogischer Unterstützung auf systemischer Ebene zur Erfüllung einer >inklusiven< Unterrichtspraxis darstellt (vgl. Abschnitt 6.1). Vor dem Hintergrund eines Generalverdachts - in Form eines als typisch angenommenen Störungsbildes - haben die Schüler*innen der Beweispflicht der Unterrichtseignung nachzukommen. In der Fokussierung auf sonderpädagogische Förderbedarfe wird dabei eine Legitimation für Aussonderung manifestiert. Inklusion wird somit zur Eigenleistung des Kindes - wer diese nicht erbringen kann, kann folglich nicht umfassend >inkludiert $<$ werden.

Auch unter Einbezug weiterer Sequenzen aus dem umfassenden Datenmaterial des Projekts StiEL, die an dieser Stelle nicht sequenzanalytisch interpretiert werden können, deutet sich ein kollektives Orientierungsmuster der Lehrkräfte an, das eine »Veranderung « (Buchner 2017) konstruiert, die kategorial entlang der Binarität von behindert/nicht-behindert operiert und nicht zwangsläufig rückgebunden ist an die tatsächliche Handlungssituation. So wird etwa am Beispiel des digitalen Klassenbuchs (vgl. Abschnitt 6.3) deutlich, wie ein entpersonalisiertes Verhaltensprofil zu Verwaltungszwecken erstellt wird, wobei die spezifischen Bedingungen und Hintergründe von Handlungen der Schüler*innen - im Sinne einer fallverstehenden pädagogischen Perspektive - keine Beachtung finden. Diese Kontrolle geschieht im Hinblick auf Inklusionsbedingungen, die sich zwar für alle gleich ausformen, deren Nicht-Erfüllung jedoch im Falle der Schüler*innen mit zugeschriebenem sonderpädagogischen Förderbedarf über eben dieses Vehikel erklärt werden kann. Dies bedeutet in der Folge, dass die dauerhaft als > die Anderen< kodierten - und damit externalisierten - keine solche systeminterne Irritation hervorrufen können, die eine Notwendigkeit zur Erwägung von Veränderungen der Praxis (z. B. eine leisere Unterrichtsgestaltung) produziert. So werden auch die schulischen Rahmenbedingungen, die letztlich die eigene Lehrpraxis entscheidend determinieren, von den Fachkräften nicht infrage gestellt (vgl. Abschnitt 6.2).

\section{Implikationen für die Entwicklung inklusionsbezogener Fort- und Weiterbildungen}

Die Bearbeitung des Desiderats einer gesättigten forschungsbasierten Rekonstruktion von Orientierungen auf Inklusion steht ebenso noch aus wie eine tiefergehende Analyse 
der schulformspezifischen Rahmenbedingungen, die an dieser Stelle nicht berücksichtigt werden kann. Die Ausweitung der Sequenzanalysen, welche an dieser Stelle nur exemplarisch betrieben werden konnten, würde dabei die Basis für eine umfängliche Fallkontrastierung und deren Weiterführung in den Abstraktionsstufen der Typenbildung herstellen. Da dies im vorliegenden Fall noch nicht möglich war, lassen sich keine generalisierbaren Ergebnisse ausweisen.

Andeutungsweise zeigen sich jedoch Anschlüsse an frühe Diskurse der Integrationsund Inklusionspädagogik, die eine schulpraktische Beibehaltung eines sogenannten $\gg$ Readiness-Modells « (Boban und Hinz 2003) kritisierten. In einem solchen Modell ist die kompensatorische Beweislast der Teilnahmefähigkeit aufseiten der Schüler*innen mit zugewiesenem Förderbedarf angesiedelt. Zudem dokumentieren sich in den Rekonstruktionen Behinderungen im Sinne eines situational-interaktionistischen Verständnisses (vgl. Sturm und Wagner-Willi 2012): als Barrieren der Gleichberechtigung vor dem Hintergrund eines leistungsnormierten Rahmens. So wird ein Inklusionsverständnis deutlich, das nicht auf Gemeinschaftlichkeit, sondern auf den individuellen Lernfortschritt einzelner Schüler*innen fokussiert. Dieses Phänomen wurde als »Sonderpädagogisierung der Inklusion « unlängst von Biermann (2019) länderübergreifend herausgearbeitet.

Darüber hinaus können erste Überlegungen zu den Anforderungen einer Professionalisierung im Kontext Inklusiver Bildung angestellt werden: Gerade, weil sich Inklusionsbedingungen einer direkten Beobachtung in der alltäglichen Handlungspraxis entziehen, erscheinen im Rahmen von Fort- und Weiterbildungen Reflexionsangebote sinnvoll, um das »Implizite explizit zu machen « (Michel 2015, 109) - und zum Beispiel Fragen von Kooperation/Multiprofessionalität systemisch und fallbasiert bearbeiten zu lassen. So haben die Rekonstruktionen aufgezeigt, dass Inklusionsbedingungen dethematisiert - und somit unhinterfragt - bleiben. Dies ist nicht verwunderlich, wird Inklusion bildungspolitisch als >Additum < zur bestehenden Schul- und Unterrichtsstruktur (Feuser 2016) bearbeitet. Dementsprechend gestaltet sich eine handlungspraktische Delegation des Abweichenden - vor dem Hintergrund einer binär-disziplinären Konstellation von Allgemeiner und Sonder-Pädagogik - als legitim (vgl. Köpfer 2018).

Weiter scheint es für die Ausgestaltung von Fort- und Weiterbildungen bedeutsam, den Rahmenbedingungen besondere Beachtung zu schenken. In der Literatur wurde hierzu bereits darauf aufmerksam gemacht, dass eine fallbasierte Gestaltung dazu geeignet ist, »die exkludierenden Aspekte von Schule und Unterricht, bzw. von pädagogischem Handeln insgesamt, reflexiv zugänglich « (Budde und Hummrich 2014, o.S.) zu machen. Eine kasuistische Reflexion vermag es dabei insbesondere, auf die oben beschriebenen Verflochtenheiten einzugehen und fallbasiert theoretische Anbindungen an den pädagogischen Integrations- und Inklusionsdiskurs, zum Beispiel im Bereich von Kooperation, Didaktik und Diagnostik, zu leisten. 
Damit würde die Professionalisierung im Bereich schulischer Inklusion wesentlich eine Beschäftigung mit Spannungsverhältnissen - im Sinne der Problematisierungen von Widersprüchen und Unvereinbarkeiten zwischen Inklusiver Bildung und gegebenen schulischen Rahmenbedingungen - sowie deren Artikulation beinhalten. Hierauf aufbauen kann das Ausloten von Spielräumen für Unterrichts- und Schulentwicklung abseits einer eindimensional auf Leistungsnormen abstellenden Perspektive (z. B. durch Optionen sozialer Partizipation zur Aushandlung von Lautstärkeregelungen). Im Rahmen der Konzeption von Fort- und Weiterbildungsangeboten kann es dabei zielführend sein, Fallbeispiele miss- und gelingender Inklusion zu beleuchten und so die Bestimmung der abgedunkelten Inklusionsbedingungen weiter zu präzisieren bzw. diese $>$ sichtbar $<$ zu machen.

\section{Anmerkungen}

1 Es wurden 134 Einzel- bzw. Gruppeninterviews mit insgesamt 142 Interviewpartner*innen geführt. Das Sampling verfolgte das Ziel, unterschiedliche (para-)professionelle Perspektiven auf die (Fortbildungs-)Bedarfe in Schulen abzubilden. Dabei wurden 26 Schulleitungen, 61 Lehrkräfte, 22 Sonderpädagog*innen, vier Schulsozialarbeiter*innen sowie sechs Schulbegleitungen an allgemein- wie berufsbildenden Schulen interviewt. Darüber hinaus zählen sechs wissenschaftliche Expert*innen, zehn Vertreter*innen von Fachverbänden sowie sieben Fortbildungsexpert*innen zu den Interviewten.

2 Um zu Aussagen über die Ausgestaltung der Praxis auf Schul- sowie auf Unterrichtsebene zu gelangen, wurden für die vertiefende Analyse ausschließlich Sequenzen aus den Interviews mit Regelschul- sowie sonderpädagogischen Lehrkräften berücksichtigt. Narrationen zeichnen sich dadurch aus, dass sich darin Ereignisse unter Nennung zeitlicher wie örtlicher Bezüge gefasst finden, und sie - im Gegensatz zu Argumentationen oder Bewertungen - einen geringen Grad an Theoretisierungen aufweisen (vgl. Nohl 2012). Im Rahmen problemzentrierter Interviews können solche narrativen Sequenzen stimuliert werden, da diese den Charakter der Offenheit (vgl. ebd.) besitzen.

3 »Veranderung « - als deutsche Übersetzung des als »Othering « bekannten Konzepts - bezieht sich auf Prozesse des >Different-machens` (vgl. Castro Varela 2016, 154).

\section{Literatur}

Aichele, Valentin und Susann Kroworsch. 2017. Inklusive Bildung ist ein Menschenrecht. Zugegriffen am 17.02.2020. https://www.institut-fuer-menschenrechte.de/fileadmin/user_upload/ Publikationen/POSITION/Position_10_Inklusive_Bildung.pdf.

Biermann, Julia. 2019 (in Vorb.). Comparing Article 24 UN CRPD's Influence on Inclusive Education in Nigeria and Germany: Institutional Change in Educational Discourses.

Boban, Ines und Andreas Hinz. 2003. Qualitätsentwicklung des Gemeinsamen Unterrichts durch den »Index für Inklusion«. Behinderte (4/5): 65-80.

Bohnsack, Ralf. 2014. Rekonstruktive Sozialforschung. 9. Auflage. Opladen: B. Budrich.

Buchner, Tobias. 2017. Markierungen und Platzierungen. Zeitschrift Für Inklusion 11 (4). Zuge- 
griffen am 17.02.2020. https//www.inklusion-online.net/index.php/inklusion-online/article/ view/435.

Budde, Jürgen. 2012. Die Rede von der Heterogenität in der Schulpädagogik. Forum qualitative Sozialforschung 13 (2). Zugegriffen am 17.02.2020. http://www.qualitative-research.net/index. php/fqs/article/viewFile/1761/3358.

Budde, Jürgen. 2018. Erziehungswissenschaftliche Perspektiven auf Inklusion und Intersektionalität. In Handbuch schulische Inklusion, hrsg. v. Tanja Sturm und Monika Wagner-Willi, 45-59. Opladen: B. Budrich.

Budde, Jürgen und Merle Hummrich. 2014. Reflexive Inklusion. Zeitschrift Für Inklusion 7 (4). Zugegriffen am 17.02.2020. https://www.inklusion-online.net/index.php/inklusion-online/article/ view/193.

Budde, Jürgen, Susanne Offen und Benjamin Heynoldt. 2014. Inklusion sichtbar machen? In Forschung zu inklusiver Bildung, hrsg. v. Michaela Lichtblau, Daniel Blömer, Ann-Kathrin Jüttner, Katja Koch und Michaela Krüger, 105-123. Bad Heilbrunn: J. Klinkhardt.

Castro Varela, María do. 2016. Postkolonialität. In Handbuch Migrationspädagogik, hrsg. v. Paul Mecheril, 152-166. Weinheim/Basel: Beltz.

Feuser, Georg. 2016. Die Integration der Inklusion in die Segregation. In Be-Hinderung der Teilhabe, hrsg. v. Ursula Böing und Andreas Köpfer, 16-28. Bad Heilbrunn: J. Klinkhardt.

Gasterstädt, Julia und Michael Urban. 2016. Einstellung zu Inklusion? Empirische Sonderpädagogik (1): 54-66.

Köpfer, Andreas. 2018. Inklusive Bildung zwischen Programmatik und Praxis - (inter-)nationale Analyse professioneller Kooperationsprozesse in inklusionsorientierten Unterrichtssettings entlang von Raum und Expertise. Bildung und Erziehung 71 (4): 395-411.

Köpfer, Andreas. 2019. Rekonstruktion behinderungsbedingter Differenzproduktion in inklusionsorientierten Schulen. In Inklusionsforschung im Spannungsfeld von Erziehungswissenschaft und Bildungspolitik, hrsg. v. Jürgen Budde, Andrea Dlugosch, Petra Herzmann, Lisa Rosen, Argyro Panagiotopoulou, Tanja Sturm und Monika Wagner-Willi, 143-164. Opladen: B. Budrich.

Krischler, Mireille, Justin J. W. Powell und Ineke M. Pit-Ten Cate. 2019. What is meant by inclusion? European Journal of Special Needs Education 34 (5): 632-648.

Mannheim, Karl. 1980. Strukturen des Denkens. Frankfurt/M.: Suhrkamp.

Michel, Burkard. 2015. Orientierungsdilemmata einer korporativen Bildpraxis. In Dokumentarische Bildinterpretation, hrsg. v. Ralf Bohnsack, Burkard Michel und Aglaja Przyborski, 107-146. Opladen: B. Budrich.

Nohl, Arnd-Michael. 2012. Interview und dokumentarische Methode. 4. Auflage. Wiesbaden: Springer.

Pfahl, Lisa. 2011. Techniken der Behinderung. Bielefeld: transcript.

Piezunka, Anne, Tina Schaffus und Michael Grosche. 2017. Vier Definitionen von schulischer Inklusion und ihr konsensueller Kern. Unterrichtswissenschaft 45 (4): 207-222.

Przyborski, Aglaja und Monika Wohlrab-Sahr. 2014. Qualitative Sozialforschung. 4. Auflage. München: Oldenbourg Verlag.

Rotter, Carolin und Rebecca Schaaf. 2015. Was verstehen Schulen unter Inklusion? In Perspektiven auf inklusive Bildung, hrsg. v. Daniel Blömer, Michaela Lichtblau, Ann-Kathrin Jüttner, Katja Koch, Michaela Krüger und Rolf Werning, 145-149. Wiesbaden: Springer.

Steiert, Viviane. 2017. Inklusion in derSchulsozialarbeit.Zugegriffen am 17.02.2020. https://duepublico2. uni-due.de/servlets/MCRFileNodeServlet/duepublico_derivate_00043513/DissVSteiert.pdf.

Sturm, Tanja und Monika Wagner-Willi. 2012. Inklusion und Milieus in schulischen Organisationen. Zeitschrift Für Inklusion 6 (4). Zugegriffen am 17.02.2020. https://www.inklusion-online.net/ index.php/inklusion-online/article/view/32. 
Sturm, Tanja und Monika Wagner-Willi, Hrsg. 2018. Handbuch schulische Inklusion. Opladen: B. Budrich.

Trescher, Hendrik. 2018. Inklusion zwischen Theorie und Lebenspraxis. Journal für Psychologie 26 (2): 29-49.

UN. 2006. Convention on the Rights of Persons with Disabilities. Zugegriffen am 17.02.2020. https:// www.un.org/development/desa/disabilities/convention-on-the-rights-of-persons-with -disabilities.html.

Wagener, Benjamin. 2018. Inklusion aus Perspektive der Praxeologischen Wissenssoziologie. In Handbuch schulische Inklusion, hrsg. v. Tanja Sturm und Monika Wagner-Willi, 77-92. Opladen: B. Budrich.

Weisser, Jan. 2005. Behinderung, Ungleichheit, Bildung. Bielefeld: transcript.

Weisser, Jan. 2017. Konfliktfelder schulischer Inklusion und Exklusion im 20. Jahrhundert. Weinheim/Basel: Beltz Juventa.

Witzel, Andreas. 2000. Das problemzentrierte Interview. Forum qualitative Sozialforschung 1 (1). Zugegriffen am 17.02.2020. http://www.qualitative-research.net/index.php/fqs/article/view/ $1132 \%$

\section{Die AutorInnen}

Andreas Köpfer, Prof. Dr., ist Juniorprofessor für Inklusive Bildung und Lernen an der Pädagogischen Hochschule Freiburg sowie Teilprojektleiter im BMBF-Projekt »StiEL - Schule tatsächlich inklusiv«.

Kontakt: andreas.koepfer@ph-freiburg.de

Katharina Papke, M. A., ist akademische Mitarbeiterin im BMBF-Projekt »StiEL - Schule tatsächlich inklusiv«.

Kontakt: katharina.papke@ph-freiburg.de

Jürgen Gerdes, Dipl. Pol., ist akademischer Mitarbeiter und Verbundkoordinator im BMBF-Projekt "StiEL - Schule tatsächlich inklusiv«.

Kontakt: juergen.gerdes@ph-freiburg.de 